\title{
INFLUENCIA DEMOGRÁFICA EN LA EVOLUCIÓN DE CARDIOPATÍAS VALVULARES CON MANEJO FARMACOLÓGICO Y DIETARIO EN PACIENTES CANINOS DE LA CIUDAD DE BUCARAMANGA Y SU ÁREA METROPOLITANA
}

\author{
Fabián Alejandro Gómez-Torres*, M.Sc. ${ }_{1}$, Favio Sánchez-Pico, Esp. ${ }_{1}$, \\ Javier Hernando Albarracín-Navas, MVZ, Esp. (c), Edgar Hernando Toledo-Cáceres, MV , \\ Oscar Mauricio Castellanos-García, Est. $\mathrm{MVZ}_{2}$
}

${ }_{1}$ Grupo de Investigación en Ciencias Animales (GRICA)

${ }_{2}$ Facultad de Medicina Veterinaria y Zootecnia

Universidad Cooperativa de Colombia, sede Bucaramanga, Colombia

\begin{abstract}
Recibido: 25 de septiembre del 2013 Aprobado: 20 de noviembre del 2013
* Autor de correspondencia: Fabián Alejandro Gómez Torres, Facultad de Medicina Veterinaria y Zootecnia, Universidad Cooperativa de Colombia, Bucaramanga, Colombia, A. A. 2019, calle 31 no․ 33A-51, oficina 602. Teléfono: 6459500 ext. 7072. Correo electrónico: fabian.gomez@campusucc.edu.co

Cómo citar este artículo: Gómez-Torres FA, Sánchez-Pico F, Albarracín-Navas JH, Toledo-Cáceres EH, Castellanos-García OM. Influencia demográfica en la evolución de cardiopatías valvulares con manejo farmacológico y dietario en caninos de la ciudad de Bucaramanga y su área metropolitana. Spei Domus. 2013; 9(19): 23-8.
\end{abstract}

Resumen. Las enfermedades cardiovasculares se presentan generalmente en perros de avanzada edad ( $>7$ años). Este es el caso de la degeneración valvular mixomatosa (DVM), que es una cardiopatía adquirida cuyo signo característico es la presencia de soplos audibles. El objetivo de esta investigación fue analizar la influencia demográfica sobre la evolución de los pacientes con tratamiento farmacológico y con dietas hiponatrémicas en DVM. Se trabajó con 60 pacientes hasta $15 \mathrm{~kg}$ de peso, hembras y machos, enteros o castrados, entre 6 y 15 años de edad, que presentaban la enfermedad en estadio B y C, que requieren medicación y soporte dietario. Se realizó una medición inicial de electrolitos, cuadro hemático, proteínas totales, densidad urinaria, creatinina y pruebas imagenológicas (radiología, ecocardiografía y electrocardiografía); posteriormente se dividieron en tres grupos con 20 pacientes cada uno y fueron medicados farmacológicamente con enalapril y espironolactona combinada (Cardial ${ }^{\circledR}$ ) y a cada uno de ellos se les asignó una dieta diferente (dieta mixta y concentrado, Hill's $\mathrm{h} / \mathrm{d}{ }^{\circledR}$ y Hill's $\mathrm{k} / \mathrm{d}{ }^{\circledR}$ ). Según los resultados, los pacientes a los que se les suministró dietas hiponatrémicas presentaron mejor comportamiento clínico y un nivel de sobrevida mayor. Debido a esto, aquellos pacientes cuyos dueños pertenecían a estratos bajos al parecer no tenían la capacidad económica de administrar a sus mascotas estas dietas, lo que repercutió en la salud de ellos, ya que el porcentaje de mortalidad fue alto (90\%).

Palabras clave: canino, cardiopatía, dieta, economía.

\section{Demographical Influence on Canine Patients in the Evolution of Valvular Cardiopathias Using Pharmacological and Dietary Handling in Bucaramanga and its Metropolitan Area}

Abstract. Cardiovascular diseases occur frequently in dogs, especially in older dogs ( $>7$ years). This is the case with myxomatous valvular degeneration (MVD), which is an acquired disease whose hallmark is the presence of audible murmurs on auscultation. The aim of this research was to analyze the demographic influence on outcomes of patients with drug treatment and hyponatraemic DVM diets. We worked with 60 patients weighing up to $15 \mathrm{~kg}$, females and males, whole or neutered, between 6-15 years of age who had the disease in stage $B$ and $C$ requiring medication and dietary support. An initial sample was taken of electrolytes $(\mathrm{Na}$, $\mathrm{Cl}$ and $\mathrm{K}$ ), blood count, total protein, urine specific gravity and creatinine, and imaging tests (radiology, echocardiography and electrocardiography) were performed; the animals were subsequently divided into

\begin{abstract}
Influência demográfica em pacientes caninos na evolução de cardiopatias valvulares com manejo farmacológico $e$ dietário em Bucaramanga e sua área metropolitana
\end{abstract}

Resumo. As doenças cardiovasculares se apresentam em cachorros de idade avançada ( $>7$ anos). Este é o caso da degeneração valvular mixomatosa (DVM), que é una cardiopatia adquirida cujo sinal característico é a presença de sopros audíveis. O objetivo desta pesquisa constitui em analisar a influência demográfica a evolução dos pacientes com tratamento farmacológico e com dietas hiponatrêmicas em DVM. Trabalhouse com 60 pacientes até $15 \mathrm{~kg}$ de peso, fêmeas e machos, inteiros ou castrados, entre 6 e 15 anos de idade, que apresentavam a doença em estágio $\mathrm{B}$ e $\mathrm{C}$, que requerem medicamento e suporte dietário. Realizou-se uma coleta inicial de eletrólitos, quadro hemático, proteínas totais, densidade urinária, creatinina e provas imagenológicas (radiologia, ecocardiografia e eletrocardiografia); posteriormente, dividiram-se em três grupos 
three study groups of 20 patients each who were pharmacologically medicated with enalapril and spironolactone (Cardial ${ }^{\circledR}$ ) and each was assigned a different diet (Mixed and concentrated Diet, Hill's H / d and Hill's $\left.{ }^{\circledR} \mathrm{K} / \mathrm{d}^{\circledR}\right)$. The results showed that patients who were given hyponatremic diets had better clinical behavior and a higher survival rate. Patients whose owners belonged to lower socioeconomic levels also lacked the economic capacity to manage their pets using these diets, which affected the health of the patients, whose mortality rate was very high (90\%).

Keywords: canine, disease, diet, economy.

\section{Introducción}

Las enfermedades cardiovasculares se presentan frecuentemente en la especie canina, sobre todo en los perros de avanzada edad (mayores de siete años) de las razas caniche, pinscher, mestizos, cavalier spaniel, principalmente. Este es el caso de la degeneración valvular mixomatosa (DVM), una cardiopatía adquirida cuyo signo característico es la presencia de soplos audibles a la auscultación, sin presencia de manifestaciones clínicas en las fases primarias de la enfermedad [1-5].

En la DVM se presenta disminución del gasto cardiaco. Esto activa mecanismos de compensación neuroendocrinos: el neurogénico, mediado por la noradrenalina, que es inmediato y a corto tiempo; el endocrino, que es la activación del sistema renina-angiotensina-aldosterona, que es prolongado en el tiempo en etapas primarias de la insuficiencia cardiaca. Cuando el fallo cardiaco en periodos de tiempo largos causa deterioros del sistema vascular, sobrecarga el sistema y se presenta elevación de la aldosterona en los pacientes con DVM, lo que ocasiona fibrosis del miocardio y variabilidad en la frecuencia cardiaca $[2,6-8]$.

El funcionamiento de las válvulas mitral y tricúspide consiste en mantenerse abiertas ampliamente durante la fase diastólica del ciclo cardiaco, lo cual permite un correcto llenado ventricular, y luego el cierre durante la fase sistólica, para evitar la regurgitación de sangre hacia las aurículas. El correcto funcionamiento de estas válvulas depende de una adecuada actividad de cada uno de los componentes que las forman - valvas, cuerdas tendinosas, músculos papilares - y cualquier alteración en alguno de ellos producirá un regreso de la sangre a las aurículas en cada ciclo cardiaco [2, 9].

Los cambios presentes en las valvas se presentan con mayor frecuencia en la válvula mitral (70-75\%), en la tricúspide (10\%) y en ambas (20-25\%), presentándose engrosamiento de los bordes libres en forma nodular, produciendo valvas opacas y rompimiento de las cuerdas tendinosas, lo que lleva a la descompensación com 20 pacientes cada um e foram medicados farmacologicamente com enalapril e espironolactona combinada (Cardial ${ }^{\circledR}$ ) e a cada um deles foi designada uma dieta diferente (Dieta mista e ração Hill's h/d ${ }^{\circledR}$ e Hill's k/ $\left.\mathrm{d}^{\circledR}\right)$. Segundo os resultados, os pacientes aos quais foram subministradas dietas hiponatrêmicas apresentaram melhor comportamento clínico e um nível de sobrevida maior. Devido a isso, aqueles pacientes cujos donos pertenciam a classes baixas ao parecer não tinham a capacidade econômica de administrar a seus animais de estimação essas dietas, o que repercutiu na saúde deles, já que a porcentagem de mortalidade foi alta $(90 \%)$.

Palavras-chave: canino, cardiopatia, dieta, economia.

del paciente con sus respectivos signos clínicos en los diferentes estadíos de la enfermedad [2, 8, 10-15].

El principal determinante del volumen de regurgitación son el tamaño del orificio donde sucede la regurgitación y el gradiente de presión sistólica entre el ventrículo y el atrio izquierdo. Se debe reconocer que la resistencia vascular sistémica, la poscarga ventricular izquierda y el estado de contractilidad cardiaca no son determinantes directos del volumen de regurgitación [16, 17].

\section{Materiales y métodos}

Esta investigación se realizó en Bucaramanga y su área metropolitana. La toma de muestras se hizo en la Clínica Veterinaria Animales de Compañía de la Universidad Cooperativa de Colombia, en pacientes a los que se les diagnosticó enfermedad valvular mixomatosa mediante ecocardiografía y otras pruebas de imagenológicas. Los propietarios diligenciaron un consentimiento informado en el que se les explicaba cómo se realizaba el trabajo de investigación.

Se trabajó con 60 pacientes de hasta $15 \mathrm{~kg}$ de peso, hembras y machos, enteros o castrados, entre los 6 y 15 años de edad, que presentaban la enfermedad en estadio $\mathrm{B}$ y C, requiriendo medicación y soporte dietario. Se realizó una toma inicial de electrolitos $(\mathrm{Na}, \mathrm{Cl}$ y K$)$, cuadro hemático, proteínas totales, densidad urinaria, creatinina y pruebas imagenológicas - radiología, ecocardiografía y electrocardiografía-. Después se dividieron en tres grupos de estudio con 20 pacientes cada uno, y fueron medicados farmacológicamente con enalapril y espironolactona combinada (Cardial ${ }^{\circledR}$ ) y a cada uno de ellos se les asignó una dieta diferente — dieta mixta y concentrado, Hill's $\mathrm{h} / \mathrm{d}^{\circledR}$ y Hill's $\mathrm{k} / \mathrm{d}^{\circledR}$-. Se hicieron pruebas trimestrales de electrolitos, creatinina y proteínas totales durante nueve meses. Se realizó un estudio prospectivo longitudinal de tipo experimental, con un componente de ensayo clínico. Se hizo un aná- 
lisis descriptivo de cada una de las variables discretas estrato social, administración de dietas prescritas- y continuas - medición de electrolitos, creatinina y proteínas totales- para cada animal y cada grupo experimental evaluado con técnicas de análisis de varianza (Anova), para determinar si existían diferencias entre grupos. Se determinó si existía alguna relación entre el nivel socioeconómico al cual pertenecían los dueños de las mascotas enfermas y la progresión de la enfermedad que finalmente llevaba al fallecimiento de los pacientes.

Tabla 1. Identificación de grupos experimentales con las dietas a utilizar y clasificados según su estrato

\begin{tabular}{|l|c|c|c|c|c|c|}
\hline Alimento/estrato & 2 & 3 & 4 & 5 & 6 & Total \\
\hline $\begin{array}{l}\text { Dieta hiponatrémica } \\
1 \text { (H/d) }\end{array}$ & 1 & 13 & 4 & 1 & 1 & 20 \\
\hline $\begin{array}{l}\text { Dieta hiponatrémica } \\
\text { 2(K/d) }\end{array}$ & 2 & 7 & 2 & 7 & 2 & 20 \\
\hline Mixta casera & 8 & 11 & 0 & 1 & 0 & 20 \\
\hline Total & 11 & 31 & 6 & 9 & 3 & \\
\hline
\end{tabular}

Fuente: elaboración propia

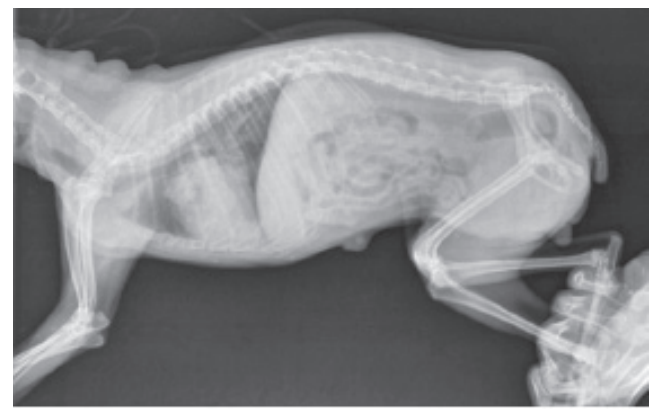

Figura 1. Paciente pinscher con cardiomegalia, edema perihiliar y elevación traqueal

Fuente: elaboración propia

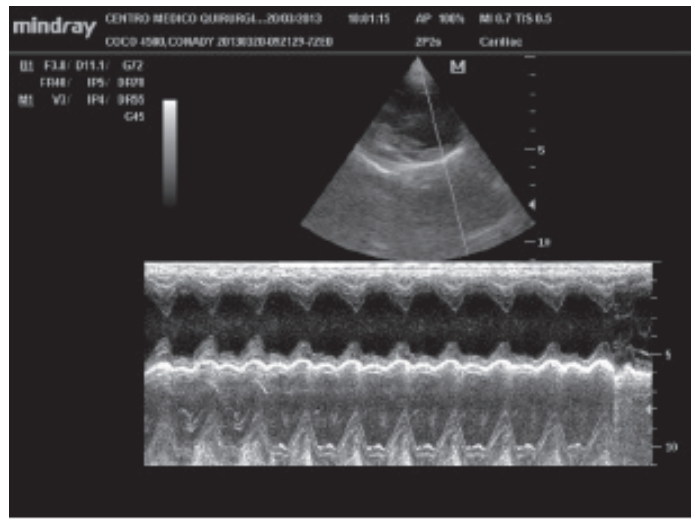

Figura 3. Ecocardiografía en modo B y M, utilizado para la medición de fracción de acortamiento para el diagnóstico de DVM Fuente: elaboración propia

\section{Resultados}

Los pacientes que ingresaron al estudio se clasificaron en el estadio B y C, según la clasificación del Colegio Americano de Medicina Interna Veterinaria. Cada paciente fue clasificado al efectuar el diligenciamiento de la historia clínica y realizar pruebas complementarias de química sanguínea e imagenología. Se encontró radiológicamente que el $87 \%$ de los pacientes presentaban índice cardiovertebral elevado, lo que indica cardiomegalia y edema perihiliar en el 100\% de los animales (figura 1).

Electrocardiográficamente, los principales tipos de arritmias encontradas fueron las supraventriculares (65\%), donde la principal de estas fue la Taquicardia Auricular Paroxística (figura 2). Los hallazgos ecocardiográficos revelaron que el 93\% de los pacientes presentaban una fracción de acortamiento mayor al $45 \%$, dicha medición sugiere que los pacientes son susceptibles a manifestar la cardiopatía. Así mismo, el 100\% de ellos presentaron regurgitación en las válvulas auriculoventriculares, lo que indica el daño en el aparato valvular (figuras 3 y 4 ).

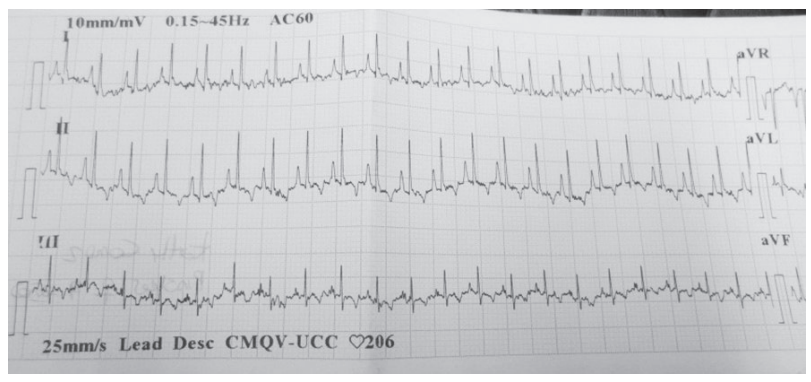

Figura 2. Electrocardiografía de un paciente que muestra Taquicardia Auricular Paroxística, con una onda P picuda Fuente: elaboración propia

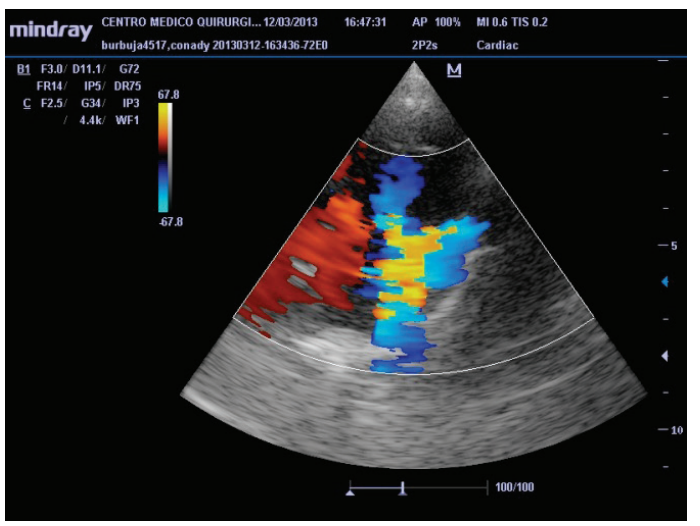

Figura 4. Ecocardiografía doppler color para reconocer los flujos turbulentos en la regurgitación mitral o tricuspidiana Fuente: elaboración propia 
Según los resultados obtenidos, los pacientes a los que se les suministraron dietas hiponatrémicas presentaron un mejor comportamiento clínico, a través de la disminución de los edemas pulmonares de origen cardiogénico, disneas y tos. Esto mostró una diferencia significativa en el nivel de sobrevida al compararlos con aquellos en los cuales su alimentación consistía en dietas caseras o concentrados comerciales, ya que en este grupo de animales el porcentaje de mortalidad fue muy alto (90\%) (tabla 2).

Al momento del segundo control se identificó un porcentaje de mortalidad del 30\% en el grupo de estudio que recibió dieta prescrita Hill's H/d (grupo 1), un 25\% correspondió al estrato medio bajo y un 5\% al estrato medio alto. En el grupo que se alimentó con Hill's K/d (grupo 2) se encontró una mortalidad del $20 \%$, y la mitad de estos animales pertenecían a un estrato medio bajo. Los animales cuya alimentación se basaba en dieta casera o concentrado comercial (grupo 3) presentaron una mortalidad del 90\% antes de obtener el segundo muestreo, y todos pertenecían a un estrato medio bajo (tabla 2).

Tabla 2. Número de animales con sobrevida al momento del segundo muestreo y el estrato al que pertenecen

\begin{tabular}{|l|c|c|c|c|c|c|}
\hline \multicolumn{1}{|c|}{ Alimento/estrato } & 2 & 3 & 4 & 5 & 6 & Total \\
\hline $\begin{array}{l}\text { Dieta hiponatrémica } \\
1(\mathrm{H} / \mathrm{d})\end{array}$ & 0 & 9 & 4 & 1 & 0 & 14 \\
\hline $\begin{array}{l}\text { Dieta hiponatrémica } \\
2(\mathrm{~K} / \mathrm{d})\end{array}$ & 1 & 6 & 1 & 6 & 2 & 16 \\
\hline Mixta casera & 1 & 1 & 0 & 0 & 0 & 2 \\
\hline Total & 2 & 16 & 5 & 7 & 2 & \\
\hline
\end{tabular}

Fuente: elaboración propia

En la tabla 3 se evidencia de manera significativa la importancia de incluir dietas hiponatrémicas en pacientes con cardiopatías, mejorando los niveles de sobrevida de estos con disminución de complicaciones secundarias por hipernatremias.

Tabla 3. Porcentaje de mortalidad de los pacientes al segundo muestreo

\begin{tabular}{|l|c|c|}
\hline \multicolumn{1}{|c|}{$\begin{array}{c}\text { Grupos/ } \\
\text { mortalidad }\end{array}$} & $\begin{array}{c}\text { Número de } \\
\text { pacientes }\end{array}$ & $\begin{array}{c}\text { Porcentaje de } \\
\text { mortalidad }\end{array}$ \\
\hline Grupo 1 (H/d) & 14 & $30 \%$ \\
\hline Grupo 2 (K/d) & 16 & $20 \%$ \\
\hline Grupo 3 (mixta) & 2 & $90 \%$ \\
\hline
\end{tabular}

Fuente: elaboración propia

En los pacientes del grupo 1, que alcanzaron el tercer muestreo, se encontró que el $60 \%$ mostró una sobrevida a la patología. En los del grupo 2 fue del 65\% y en los del grupo 3 sólo el 10\% logró sobrevivir a los efectos secundarios de la cardiopatía (tabla 4).

De los pacientes que sobrevivieron hasta el final del estudio del grupo 1, el 58\% pertenecían al estrato medio bajo y el $42 \%$ al estrato medio alto. De los que sobrevivieron en el grupo 2, el 38\% pertenecían al estrato medio bajo y el $62 \%$ al estrato medio alto (tablas 4 y 5 ).

Tabla 4. Número de animales con sobrevida al momento del tercer muestreo y el estrato al que pertenecen

\begin{tabular}{|l|l|l|l|l|l|l|}
\hline \multicolumn{1}{|c|}{ Alimento/estrato } & $\mathbf{2}$ & $\mathbf{3}$ & $\mathbf{4}$ & $\mathbf{5}$ & $\mathbf{6}$ & Total \\
\hline Dieta hiponatremica 1 (H/d) & 0 & 7 & 4 & 1 & 0 & 12 \\
\hline Dieta hiponatremica 2 (K/d) & 1 & 4 & 1 & 5 & 2 & 13 \\
\hline Mixta casera & 1 & 1 & 0 & 0 & 0 & 2 \\
\hline Total & 2 & 12 & 5 & 6 & 2 & \\
\hline
\end{tabular}

Fuente: elaboración propia

Tabla 5. Porcentaje de sobrevida de los pacientes al tercer muestreo

\begin{tabular}{|l|l|l|}
\hline \multicolumn{1}{|c|}{$\begin{array}{c}\text { Grupos/ } \\
\text { mortalidad }\end{array}$} & \multicolumn{1}{|c|}{$\begin{array}{c}\text { Número de } \\
\text { pacientes }\end{array}$} & \multicolumn{1}{c|}{$\begin{array}{c}\text { Porcentaje de } \\
\text { sobrevida }\end{array}$} \\
\hline Grupo 1 (H/d) & 12 & $60 \%$ \\
\hline Grupo 2 (K/d) & 13 & $65 \%$ \\
\hline Grupo 3 (mixta) & 2 & $10 \%$ \\
\hline
\end{tabular}

Fuente: elaboración propia

\section{Discusión de resultados}

Los cambios radiológicos encontrados en este estudio coinciden con la mayoría de los reportes, donde se especifica en forma detallada cada una de las alteraciones presentadas en la anatomía radiológica, como agrandamiento de cámaras, elevaciones traqueales, entre otras [18-20].

En esta investigación se observó una alta presentación de arritmias supraventriculares en los pacientes diagnosticados con DVM, lo que no muestra un soporte en los reportes previos, ya que indican que los hallazgos electrocardiográficos no son específicos [18].

Los daños del aparato valvular y la afección de la contractilidad cardíaca diagnosticada ecográficamente coinciden con estudios anteriores, en los que se indica que la ecocardiografía es el método diagnóstico no invasivo de elección para detectar las lesiones valvulares, en el que podemos evaluar la gravedad de la insuficiencia y, finalmente, también permite evaluar su impacto en la remodelación cardiaca, la función miocárdica, la presión de llenado de los ventrículos y la presión de la arteria pulmonar [9, 21-23].

Según los resultados obtenidos, se nota la importancia de la inclusión de dietas hiponatrémicas en el 
manejo clínico de pacientes con cardiopatía a mediano y a largo plazo, debido a que se aumenta el tiempo de sobrevida de los animales afectados por la patología. Además, la literatura evidencia que la retención de sodio aporta a la presentación de edemas y por tanto la restricción de electrolitos en la dieta debe usarse en el manejo del fallo cardiaco [24].

Observando el comportamiento del nivel de sobrevida de los pacientes después de los tres seguimientos realizados, se puede concluir que los animales que pertenecían a un estrato medio alto, y cuyos propietarios tenían un mayor poder adquisitivo para mantener el tratamiento farmacológico y dietario durante el desarrollo de la enfermedad, lograron mantener un estado clínico estable que les permitió la sobrevivencia durante un tiempo más prolongado frente a aquellos pacientes a los que sus propietarios no lograron administrarles el tratamiento, debido a que su bajo nivel económico no permitía la compra de los medicamentos y dietas prescritas de forma constante, para así disminuir la velocidad de progresión de la patología.

\section{Referencias}

[1] Ware WA. Acquired valve disease. Cardiovascular disease in small animal medicine. Londres: Manson Publishing; 2007. 263-279.

[2] Gómez L. Degenerative valve disease in dogs: update on diagnosis, treatment and prognosis. Rev Colomb Cienc Pecu. Abr-Jun 2011; 24(2): 201-208.

[3] Whitney JC. Cardiovascular pathology. J Small Anim Pract. 1967; 8(8): 459-465.

[4] Buchaman JW. Chronic valvular disease (endocardiosis) in dogs. Adv Vet Sci Comp Med. 1977; 21: 75-106.

[5] Borgarelli M, Buchanan JW. Historical review, epidemiology and natural history of degenerative mitral valve disease. J Vet Cardiol. Mar 2012; 14(1): 93-101.

[6] Chatterjee K. Neurohormonal activation in congestive heart failure and the role of vasopressin. Am J Cardiol. May 2005; 95(9A):8B-13B.

[7] Jeunesse MS, Woehrle F, Scheneider M, Pharm D, Lefebvre HP. Effect of spironolactone on diuresis and urine sodium and potassium excretion in healthy dogs. J Vet Cardiol. Nov 2007; 9(2): 63-8.

[8] Dillon AR, Dell'Italia LJ, Tilson M, Killingsworth C, Denney T, Hathcock J, et al. Left ventricular remodeling in preclinical experimental mitral regurgitation of dogs. J Vet Cardiol. 2012; 14(1): 73-92.

[9] Mucha CJ. Degeneración valvular mixomatosa. REDVET. Jul 2007; 8(7): 1-7.
[10] Kogure K. Pathology of chronic mitral valvular disease in the dog. Nippon Juigaku Zasshi. 1980; 42(3): 323-35.

[11] Atkins C, Keene B, Brown W, Coats J, Crawford M, DeFrancesco $\mathrm{T}$, et al. Results of the veterinary enalapril trial to prove reduction in onset of heart failure in dogs chronically treated with enalapril alone for compensated, naturally occurring mitral valve insufficiency. J Vet Intern Med. Oct 2007; 231(7): 1061-9.

[12] Atkins C, Bonagura J, Ettinger S, Fox P, Gordon S, Haggstrom J, et al. Guidelines for the diagnosis and treatment of canine chronic valvular heart disease. J Vet Inter Med. Nov-Dec 2009; 23(6): 1142-50.

[13] Bretschneider J. Pathologie und pathogenese der sogenannten endocarditis valvularis chronica fibrosa des hundes. Diss, Universitat Giessen, Giessne. 1962.

[14] Das KM, Tashjian RJ. Chronic mitral valve disease in the dog. Vet Med Small Anim Clin. 1965; 60(12): 1209-16.

[15] Detweiler DK, Patterson DF. The prevalence and types of cardiovascular disease in dogs. Ann N Y Acad Sci. Sep 1965; 127(1): 481-516.

[16] Gaasch WH, Aurigemma GP. Inhibition of the reninangiotensin system and the left ventricular adaptation to mitral regurgitation. J Am Coll Cardiol. Abr 2002; 39(8): 1380-3.

[17] Gaasch WH, Meyer TE. Left ventricular response to mitral regurgitation: implications for management. Circulation. Nov 2008; 118(22): 2298-303.

[18] Pedersen HD, Häggström J. Mitral valve prolapsed in the dog: a model of mitral valve prolapse in man. Cardiovasc Res. 2000; 47(2): 234-43.

[19] Buchaman JW, Bucheler J. Vertebral scale system to measure canine Heart size in radiographs. J Am Vet Med Assoc. Ene 1995; 206(9): 194-9.

[20] Reyes JP, Gómez FA, Sanchéz F, Albarracín JH, Toledo $\mathrm{EH}$. Myxomatous valve degeneration a look at the latest developments of disease. Spei Domus. 2013; 9(18): 49-58.

[21] Chetboul V, Tissier R. Echocardiographic assessment of canine degenerative mitral valve disease. J Veterinary Cardiol. Mar 2012; 14(1): 127-48.

[22] Griffiths LG, Fransioli JR, Chigerwe M. Echocardiographic assessment of interventricular and intraventricular mechanical synchrony in normal dogs. J Vet Cardiol. Jun 2011; 13(2): 115-26.

[23] Hezzell MJ, Boswood A, Moonarmart W, Elliott J. Selected echocardiographic variables change more rapidly in dogs that die from Myxomatous mitral valve disease. J Vet Cardiol. Mar 2012; 14(1): 269-79.

[24] Atkins CE. Advances in the Management of Canine Heart Failure. Proceedings of the Congreso Latinoamericano de Emergencia y Cuidados Intensivos LAveccs. 2010. 